\title{
Tradução para o português brasileiro e adaptação transcultural do NCCPC-PV para avaliação de dor em pacientes com incapacidade intelectual de comunicação*
}

\section{Translation into Brazilian Portuguese and Cross-Cultural Adaptation of the NCCPC-PV for Pain Evaluation of Patients with Intellectual Disability to Communicate}

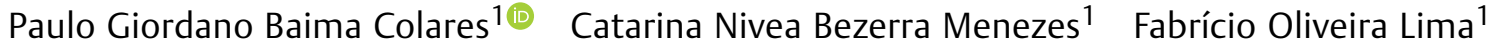 \\ Viviane Rocha Celedonio ${ }^{1}$ Lara Moreira Teles de Vasconcelos ${ }^{1}$ Josenilia Maria Alves Gomes ${ }^{1}$ \\ ${ }^{1}$ Universidade de Fortaleza (UNIFOR), Fortaleza, CE, Brazil \\ Endereço para correspondência Paulo Giordano Baima Colares, \\ Master, Universidade de Fortaleza (UNIFOR), Avenida Washington \\ Rev Bras Ortop 2020;55(2):170-180. \\ Soares 1.321, Edson Queiroz, Fortaleza, CE, 60811-905, Brasil \\ (e-mail: paulocolares@me.com).
}

\section{Resumo \\ Palavras-chave \\ - dor \\ - deficiência intelectual \\ - medição de dor}

Objetivo Realizar a tradução e a adaptação transcultural para o português falado no Brasil do instrumento "Non-Communicating Children's Pain Checklist - Postoperative Version" (NCCPC-PV), destinado a avaliar a dor aguda em indivíduos com deficiência intelectual (DI) grave que apresentam grande comprometimento cognitivo e incapacidade de comunicação (CCIC).

Método No processo de adaptação utilizado, o NCCPC-PV original foi traduzido, retraduzido, suas versões foram discutidas por um comitê de especialistas, e a ferramenta resultante foi testada em 20 profissionais de saúde e 20 cuidadores de pacientes com CCIC quanto à sua clareza semântica.

Resultados Os dados deste estudo e de seus participantes foram analisados, e seus resultados foram descritos. Dessa maneira, obteve-se a Lista de Verificação de Dor em Crianças Não Comunicantes - Versão Pós-operatória (Br-NCCPC-PV) como a versão final para o português falado no Brasil.

Conclusão Após este estudo, a Br-NCCPC-PV foi considerada adequada para o uso na população brasileira.

Abstract
Objective To perform the translation and cross-cultural adaptation to Brazilian Portuguese of the Non-Communicating Children's Pain Checklist - Postoperative Version (NCCPC-PV) instrument, which assesses acute pain in individuals with severe

\footnotetext{
Estudo realizado no Programa de Pós-Graduação em Ciências Médicas da Universidade de Fortaleza (UNIFOR), Fortaleza, CE, Brasil.
}

recebido

02 de Abril de 2018

aceito

08 de Janeiro de 2019
DOI https://doi.org/

10.1055/s-0039-3400739. ISSN 0102-3616.
Copyright $(2020$ by Sociedade Brasileira License terms de Ortopedia e Traumatologia. Published by Thieme Revinter Publicações Ltda, Rio de Janeiro, Brazil 


\section{Keywords}

- pain

- intellectual disability

- pain measurement intellectual disability (ID) who present great cognitive impairment and inability to communicate (CIIC).

Method In the adaptation process, the original NCCPC-PV was translated, backtranslated, its versions were discussed by a committee of experts, and the resulting tool was tested in 20 health professionals and 20 caregivers of $\mathrm{CCIC}$ patients regarding its semantic clarity.

Results Data from the present study and its participants were analyzed and their results were described. Thus, "Lista de Verificação de Dor em Crianças Não Comunicantes - Versão Pós-operatória” (Br-NCCPC-PV) was obtained as the final version in Brazilian Portuguese.

Conclusion After the present study, the Br-NCCPC-PV was considered adequate for use in the Brazilian population.

\section{Introdução}

A Organização Mundial da Saúde (OMS) define a deficiência intelectual (DI) como um estado da mente que apresenta o desenvolvimento incompleto ou interrompido das habilidades que contribuem para o nível de inteligência, como as capacidades cognitiva, de linguagem, e de interação social. ${ }^{1}$

A prevalência geral de DI é de $1,03 \%$, sendo quase duas vezes maior nos países subdesenvolvidos se comparada com países de alta renda. ${ }^{2}$ Petterson et al, ${ }^{3}$ encontraram prevalência de $1 \%$ de DI na população geral, mas uma prevalência oito vezes maior em se tratando de crianças que apresentavam comorbidades ao nascimento. Um estudo de autoria de van Schrojenstein Lantman-De Valk et al ${ }^{4}$ revelou que o risco de comorbidades era 2,5 vezes maior para as pessoas com DI do que para aquelas sem DI. O diagnóstico dessas doenças simultâneas entre pacientes com DI pode ser difícil, principalmente nos casos mais severos, devido à falta de ferramentas apropriadas e de profissionais treinados para identificar tais condições nesses indivíduos com comprometimento cognitivo e incapacidade de comunicação (CCIC). ${ }^{5}$

Além das comorbidades, pacientes com CCIC têm capacidade reduzida de expressar suas preocupações com a própria saúde, proporcionando uma visão limitada das suas necessidades. Observou-se que os pacientes com CCIC sofrem mais acidentes frequentemente associados a dor e desconforto; entretanto, sua dor nem sempre é prontamente reconhecida, e, sendo mal avaliada, pode ser manejada inadequadamente ou não tratada. ${ }^{6-8} \mathrm{~A}$ gravidade dessa situação evidencia a necessidade de se desenvolver melhores estratégias de manejo clínico, podendo, assim, alcançar reduções substanciais da dor, melhora da qualidade de vida, e melhores resultados em longo prazo. ${ }^{7} \mathrm{~A}$ identificação desses fatores de risco para etiologias de dor específicas pode ajudar cuidadores e profissionais. ${ }^{9}$

Esses indivíduos com DI grave estão em risco porque frequentemente apresentam condições médicas que podem causar dor, muitas vezes requerendo procedimentos, cirúrgicos ou não, que podem também ser potencialmente dolorosos. Muitos têm comportamentos idiossincráticos, que podem mascarar a expressão de dor, sendo assim difíceis de interpretar. ${ }^{10}$

Diante da escassez de instrumentos para avaliação de dor aguda em pacientes com CCIC, Breau et a ${ }^{11}$ desenvolveram e validaram o instrumento "Non-communicating Children's Pain Checklist - Postoperative Version" (NCCPC-PV), utilizado para quantificar a dor após procedimentos cirúrgicos ou devido a procedimentos que possam provocar dor aguda realizados em outros ambientes.

No Brasil, país em que a prevalência de DI é de $0,8 \%$, e em que $54,8 \%$ dos casos são graves, não há instrumentos desenvolvidos para avaliar a dor aguda em pacientes com CCIC, mesmo em condições pós-operatórias. ${ }^{12}$ Portanto, o presente estudo visa descrever a tradução e adaptação transcultural do instrumento NCCPC-PV para o português falado no Brasil, que, após validado, poderá ser usado com segurança em diversos cenários clínicos, facilitando e otimizando condutas analgésicas para esse tipo específico de pacientes.

\section{Metodologia}

Este foi um estudo do tipo observacional, transversal e descritivo. 0 processo utilizado ( - Figura 1 ) foi composto de seis fases, de acordo com as orientações para adaptação transcultural de instrumentos de medidas em saúde descritas por Guillemin et al ${ }^{12}$ e modificadas por Beaton et al. ${ }^{13}$

Durante a primeira fase (1), foi realizada a tradução do instrumento original em inglês para o português falado no Brasil por dois tradutores independentes sem conhecimento prévio do instrumento. Os tradutores envolvidos eram dois brasileiros natos bilíngues, sendo um médico, e o outro, tradutor profissional, que chegaram a uma versão final de consenso chamada Síntese da Tradução 1,2 (T1,2).

Na segunda etapa (2), foi realizada a retradução da T1,2 novamente para o inglês, por dois tradutores, de maneira autônoma, independente e cega quanto ao instrumento original. Os tradutores escolhidos tinham o inglês como língua materna, e não eram médicos ou de qualquer área da saúde. Após produzirem as suas versões das retraduções, chamadas RT1 e RT2, sintetizaram uma versão designada Síntese da Retradução 1,2 (RT1,2).

Durante a terceira etapa (3), foi realizada a avaliação da versão original do instrumento, assim como da T1, T2, T1,2, RT1, RT2 e RT1,2, por um comitê de especialistas (CE), que produziu uma versão pré-final. Esse comitê foi composto por 


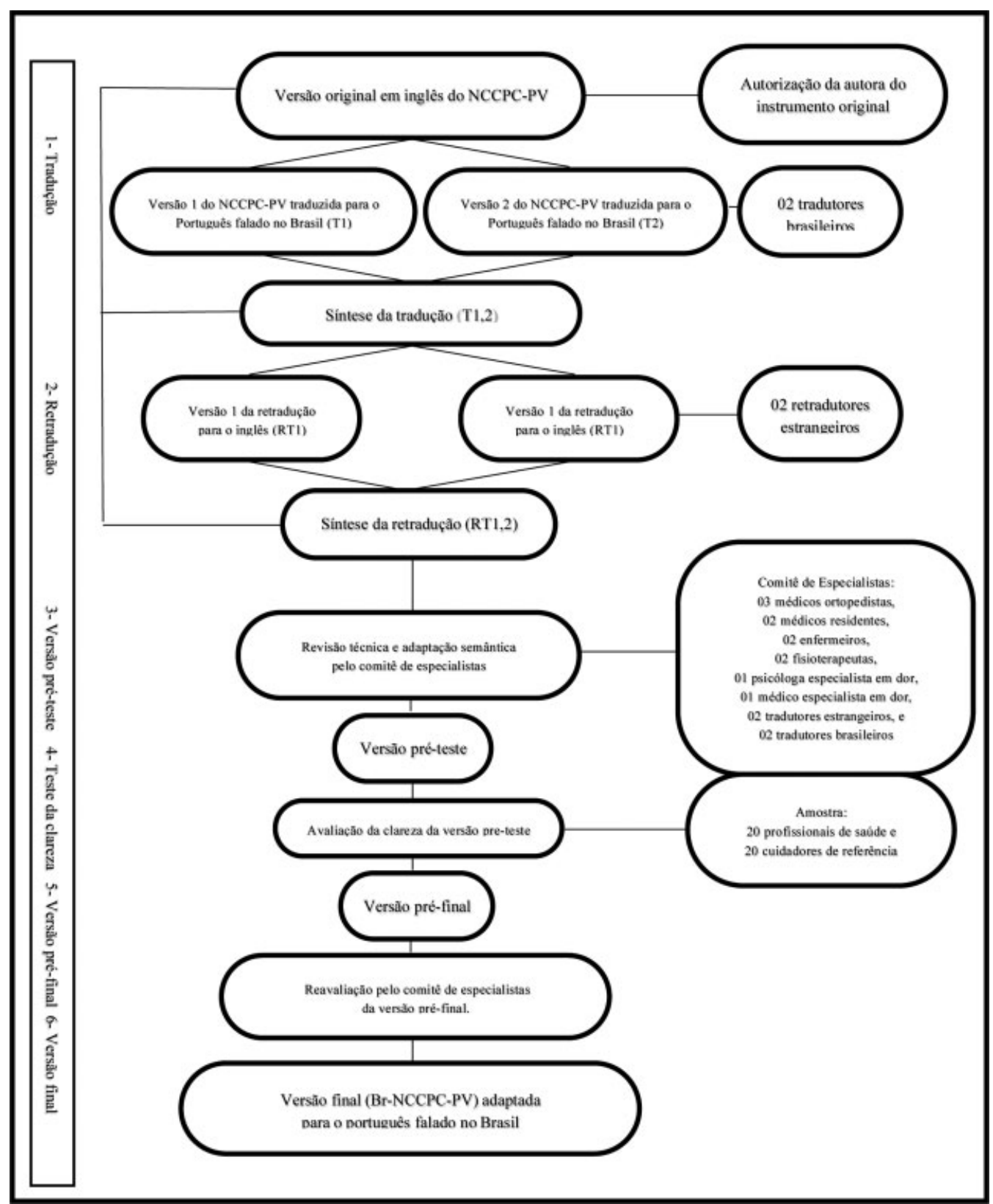

Fig. 1 Etapas do processo de adaptação.

dez profissionais de saúde interdisciplinar envolvidos no atendimento de pacientes com CCIC, uma psicóloga com experiência em processos de adaptações transculturais e validações de instrumentos de qualidade de vida, e pelos quatro tradutores envolvidos nas etapas anteriores. $\mathrm{O}$ objetivo foi produzir a versão pré-teste.

Por conseguinte, a quarta etapa (4) avaliou a clareza dos termos da versão pré-teste em uma amostra da população alvo, ou seja, 20 profissionais de saúde e 20 cuidadores, utilizando uma escala Likert com as mesmas 5 possibilidades: não claro; pouco claro; nem muito e nem pouco claro; claro; e muito claro. Os dados foram coletados nos períodos de maio de 2017 a setembro de 2017. No grupo dos profissionais de saúde, foram incluídos profissionais com formação acadêmica formal concluída em alguma área da saúde, e com experiência no atendimento de crianças com CCIC, sobretudo no manejo de situações dolorosas. No grupo dos cuidadores, foram incluídos cuidadores de crianças que apresentam CCIC que são atendidas nos ambulatórios de Ortopedia Pediátrica e/ou Reabilitação das instituições envolvidas. Foram considerados inaptos para participar da amostra cuidadores de pacientes com capacidade de autorrelato para queixa de dor e incapacidade, e cuidadores incapazes de entender todos os processos da pesquisa. Para fins epidemiológicos, foram utilizadas questões relativas à atividade prática e à experiência dos cuidadores com os pacientes com CCIC.

A versão pré-final foi definida durante a quinta etapa (5). As respostas dos dois grupos foram avaliadas separadamente e conjuntamente, e suas medianas foram calculadas a fim de identificar os itens com menor clareza. Devido ao pequeno tamanho da amostra, foram utilizadas estatísticas não paramétricas. As variáveis contínuas foram descritas como medianas e intervalos interquartil (IIQs). As variáveis categóricas 
foram descritas como números absolutos e porcentagens. Os profissionais de saúde e cuidadores foram comparados quanto a idade, sexo, escolaridade (Ensino Fundamental, Ensino Médio e Ensino Superior), e procedência (bairros da cidade onde a pesquisa foi realizada). Para a comparação de variáveis contínuas, foi utilizado teste de Mann-Whitney, e para a comparação de variáveis categóricas, foram utilizados o teste exato de Fisher ou o teste do qui-quadrado conforme apropriado. Todas as análises foram realizadas com o programa Statistical Package for the Social Sciences (SPSS, IBM Corp., Armonk, NY, EUA), versão 20.0. Valores de $p<0,05$ foram considerados estatisticamente significativos.

Na sexta etapa (6), os itens considerados menos claros foram novamente avaliados somente com os cuidadores, por esses terem obtidos medianas menores. Os seis itens menos claros tiveram uma nova questão norteadora: "Como este item poderia ficar mais claro?". Suas respostas abertas foram compiladas, e as sugestões dadas pelos cuidadores abordados foram rediscutidas com o $\mathrm{CE}$, sendo obtida a versão final.

A tradução foi previamente autorizada pela autora do instrumento NCCPC-PV original. Este estudo teve a aprovação do comitê de ética em pesquisa (CEP), estando todos os princípios éticos envolvidos na pesquisa em seres humanos no Brasil conforme a resolução $\mathrm{n}^{\circ}$ 466/2012 do Conselho Nacional de Saúde. Todos os integrantes dos grupos amostrais assinaram o termo de consentimento livre e esclarecido, e participaram da pesquisa de forma voluntária.

\section{Resultados}

A primeira etapa foi constituída da tradução para o português brasileiro do documento original NCCPC-PV em inglês. Todos os termos traduzidos (versões T1, T2 e T1,2) nessa fase são apresentados na - Tabela 1.

Na segunda etapa, foram produzidas duas versões individuais da retradução da versão (T1,2) novamente para o inglês, e então uma versão conjunta (RT1,2) foi sintetizada. A - Tabela 2 mostra todos os termos retraduzidos independentemente, bem como a versão síntese da retradução (RT1,2).

Durante a terceira etapa, após a avaliação de todas as versões pelo $C E$, foi produzida a versão pré-teste, que foi avaliada durante a quarta etapa. Um total de 40 indivíduos analisaram a clareza de todos os 27 itens, e a versão pré-teste foi usada para avaliar 20 pacientes de referência.

Quantos aos dados demográficos, as crianças tinham em média 3,09 (9-84 meses) anos, e a paralisia cerebral (PC) por anoxia cerebral foi o diagnóstico mais encontrado, com 9 (45\%) dos casos. A microcefalia e a síndrome de Down também tiveram um número maior de casos, com 4 (20\%) cada. A - Tabela 3 ilustra as características epidemiológicas dos pacientes de referência.

Todos os profissionais de saúde envolvidos tinham mais de 10 anos de formação em suas profissões, 14 (70\%) tinham mais de 10 anos de vivência com pacientes com CCIC, e 14 (70\%) trabalhavam em hospitais públicos. Os dados estão descritos na - Tabela 4.

Observou-se no grupo dos cuidadores (-Tabela 5) que a mãe era o principal cuidador do paciente (17 [85\%] casos), a atividade principal exercida era a de dona de casa (15 [75\%] casos) e que os cuidadores permaneciam entre 12 a 18 horas ( 7 [35\%] casos) ou mais do que 18 horas (11 [55\%] casos) por dia na presença da criança de referência.

A - Tabela 6 mostra as medianas da avaliação de cada um dos 27 itens que compõem a versão pré-final do questionário.

A - Tabela 7 correlaciona algumas características dos cuidadores e dos profissionais de saúde. Encontrou-se que a mediana das idades dos profissionais de saúde foi de 10 anos a mais do que a dos cuidadores. 0 sexo feminino foi mais presente, mas não houve diferença significativa entre os dois grupos. Encontrou-se diferença significativa com relação à educação, pois os profissionais de saúde em geral tinham maior grau de instrução.

Durante a avaliação das medianas e percentis da versão pré-final, foram identificados 6 itens $(9,10,11,12,16$ e 24) com medianas de até 3,5; ou seja, classificados como "nem muito e nem pouco claro" pela escala Likert utilizada. Essas classificações piores foram encontradas somente entre os cuidadores e, por esse motivo, eles foram escolhidos para um novo teste por meio de um questionário aberto, a fim de tentar realizar os últimos melhoramentos e ajustes quanto à clareza.

Primeiramente, o item 9 da versão pré-final, “testa franzida”, foi ajustado segundo sugestão do CE para "testa franzida, com o rosto tenso" para melhor correlação com avaliações álgicas.

No item 10, o termo "squinting of eyes" foi traduzido como “olhos estrábicos”, e foi pouco compreendido. Após rediscussão, decidiu-se que a melhor tradução para "squinting of eyes" seria "olhos apertados", assim ficou esse item na versão final. Outro termo sugerido para "squinting of eyes" foi "olhos apertados, fixados, ou assustados".

Já o item 11, "virando a boca para baixo, sem sorrir", após breve discussão, foi alterado para "virando a boca para baixo, sem sorrir, fazendo beicinho". Para o item 12, todas as sugestões foram recusadas pelo CE, ficando o item como já tinha sido definido na versão pré-teste.

O item 16 foi o que mais gerou discussão: "flácido". Diversas sugestões foram apresentadas pelos cuidadores, como "com o músculo mole", "musculatura mole", "corpo relaxado", e "molinho." Ao final, a proposta aceita foi "flácido, com o corpo relaxado."

Por fim, o item 24, "sudorese, transpiração", foi alterado para "sudorese, suando muito, transpirando."

Outras modificações também foram sugeridas. No item 1, o termo "whimpering" retornou para a tradução "choramingando", e o termo "whining" foi trocado para "reclamando". No item 3, o termo "screaming" mudou para "gritando". No item 13 , o termo "chewing", que anteriormente tinha sido traduzido como "mordendo", foi trocado para "mastigando." No item 20 , a tradução final ficou "encolhendo ou recolhendo a parte do corpo que se encontra sensível ao toque". Por fim, o termo "gasping", do item 26, foi traduzido como "ofegante".

Assim, chegou-se à versão final da adaptação transcultural para o português falado no Brasil denominada Lista de Verificação de Dor em Crianças Não Comunicantes - Versão Pós-Operatória (Br-NCCPC-PV), que, por fim, foi considerada adaptada. 
174 Tradução para português brasileiro e adaptação transcultural do NCCPC-PV Colares et al.

Tabela 1 Descrição da fase de tradução do NCCPC-PV original

\begin{tabular}{|c|c|c|c|c|}
\hline & Escala original & Tradução T1 & Tradução T2 & Versão T1,2 \\
\hline 1 & $\begin{array}{l}\text { Moaning, whining, whimpering } \\
\text { (fairly soft) }\end{array}$ & $\begin{array}{l}\text { Gemendo, choramingando, sol- } \\
\text { uçando (levemente) }\end{array}$ & $\begin{array}{l}\text { Gemendo, choramingando, solu- } \\
\text { çando (levemente) }\end{array}$ & $\begin{array}{l}\text { Gemendo, choramingando, solu- } \\
\text { çando (levemente) }\end{array}$ \\
\hline 2 & Crying (moderately loud) & $\begin{array}{l}\text { Chorando (moderadamente } \\
\text { alto) }\end{array}$ & Chorando (moderadamente alto) & Chorando (moderadamente alto) \\
\hline 3 & Screaming/yelling (very loud) & Chorando/berrando (muito alto) & Gritando/ Berrando (muito alto) & Gritando/berrando (muito alto) \\
\hline 4 & $\begin{array}{l}\text { A specific sound or word for pain } \\
\text { (e.g., a word, cry or type of laugh) }\end{array}$ & $\begin{array}{l}\text { Um som ou palavra específica } \\
\text { para a dor (p. ex., choro ou tipo } \\
\text { de risada) }\end{array}$ & $\begin{array}{l}\text { Um som ou palavra especifica para } \\
\text { dor (p. ex., uma palavra, grito ou } \\
\text { tipo de sorriso) }\end{array}$ & $\begin{array}{l}\text { Um som ou palavra específica para } \\
\text { a dor (p. ex., choro ou tipo de } \\
\text { risada) }\end{array}$ \\
\hline 5 & $\begin{array}{l}\text { Not cooperating, cranky, irritable, } \\
\text { unhappy }\end{array}$ & $\begin{array}{l}\text { Não cooperativa, mal-humor- } \\
\text { ada, irritadiça, triste }\end{array}$ & Não cooperativa, irritadiça, triste & $\begin{array}{l}\text { Não cooperativa, mal-humorada, } \\
\text { irritadiça, triste }\end{array}$ \\
\hline 6 & $\begin{array}{l}\text { Less interaction with others, } \\
\text { withdrawn }\end{array}$ & $\begin{array}{l}\text { Menos interativa com os outros, } \\
\text { retraída }\end{array}$ & $\begin{array}{l}\text { Menos interativo com outras } \\
\text { pessoas, retraído }\end{array}$ & $\begin{array}{l}\text { Menos interativa com os outros, } \\
\text { retraída }\end{array}$ \\
\hline 7 & $\begin{array}{l}\text { Seeking comfort or physical } \\
\text { closeness }\end{array}$ & $\begin{array}{l}\text { Buscando conforto ou } \\
\text { proximidade física }\end{array}$ & $\begin{array}{l}\text { Procurando por conforto ou } \\
\text { aproximação física }\end{array}$ & $\begin{array}{l}\text { Buscando conforto ou proximidade } \\
\text { física }\end{array}$ \\
\hline 8 & $\begin{array}{l}\text { Being difficult to distract, not able } \\
\text { to satisfy or pacify }\end{array}$ & $\begin{array}{l}\text { Difícil de distrair, incapaz de ser } \\
\text { satisfeita ou acalmada }\end{array}$ & $\begin{array}{l}\text { Difícil de distrair, incapaz de ser } \\
\text { satisfeita ou acalmada }\end{array}$ & $\begin{array}{l}\text { Difícil de distrair, incapaz de ser } \\
\text { satisfeita ou acalmada }\end{array}$ \\
\hline 9 & A furrowed brow & Testa franzida & Sobrancelha enrugada & Testa franzida \\
\hline 10 & $\begin{array}{l}\text { A change in eyes, including: } \\
\text { squinting of eyes, eyes opened } \\
\text { wide, eyes frowning }\end{array}$ & $\begin{array}{l}\text { Uma alteração nos olhos, } \\
\text { incluindo: olhos estrábicos, } \\
\text { olhos arregalados, olhos } \\
\text { franzidos }\end{array}$ & $\begin{array}{l}\text { Uma alteração dos olhos, incluindo } \\
\text { olhos estrábicos, olhos arregalados, } \\
\text { olhos carrancudos }\end{array}$ & $\begin{array}{l}\text { Uma alteração nos olhos, incluindo: } \\
\text { olhos estrábicos (apertados), olhos } \\
\text { arregalados, olhos franzidos } \\
\text { (semiabertos) }\end{array}$ \\
\hline 11 & $\begin{array}{l}\text { Turning down of mouth, not } \\
\text { smiling }\end{array}$ & $\begin{array}{l}\text { Virando a boca para baixo, sem } \\
\text { sorrir }\end{array}$ & $\begin{array}{l}\text { Boca direcionada para baixo, sem } \\
\text { sorrir }\end{array}$ & $\begin{array}{l}\text { Virando a boca para baixo, sem } \\
\text { sorrir }\end{array}$ \\
\hline 12 & $\begin{array}{l}\text { Lips puckering up, tight, pouting, } \\
\text { or quivering }\end{array}$ & $\begin{array}{l}\text { Lábios cerrados, apertados, } \\
\text { fazendo biquinho ou tremendo }\end{array}$ & $\begin{array}{l}\text { Lábios franzidos, cerrados, amua- } \\
\text { dos ou tremendo }\end{array}$ & $\begin{array}{l}\text { Lábios cerrados, apertados, } \\
\text { fazendo biquinho ou tremendo }\end{array}$ \\
\hline 13 & $\begin{array}{l}\text { Clenching or grinding teeth, } \\
\text { chewing or thrusting tongue out }\end{array}$ & $\begin{array}{l}\text { Dentes cerrados ou rangendo, } \\
\text { mordendo ou pondo a língua } \\
\text { para fora }\end{array}$ & $\begin{array}{l}\text { Dentes cerrados ou rangendo, } \\
\text { mordendo a língua ou empurrando- } \\
\text { a para fora }\end{array}$ & $\begin{array}{l}\text { Dentes cerrados ou rangendo, } \\
\text { mordendo ou pondo a língua para } \\
\text { fora }\end{array}$ \\
\hline 14 & Not moving, less active, quiet & $\begin{array}{l}\text { Sem se movimentar, menos } \\
\text { ativa, quieta }\end{array}$ & $\begin{array}{l}\text { Sem movimentar-se, menos ativa, } \\
\text { quieta }\end{array}$ & $\begin{array}{l}\text { Sem se movimentar, menos ativa, } \\
\text { quieta }\end{array}$ \\
\hline 15 & Jumping around, agitated, fidgety & Debatendo-se, agitada, inquieta & Debatendo-se, agitada, inquieta & Debatendo-se, agitada, inquieta \\
\hline 16 & Floppy & Flácido & Flácido & Flácido \\
\hline 17 & Stiff, spastic, tense, rigid & Teso, espástico, tenso, rígido & Espástico, tenso, rígido & Teso, espástico, tenso, rígido \\
\hline 18 & $\begin{array}{l}\text { Gesturing to or touching part of } \\
\text { the body that hurts }\end{array}$ & $\begin{array}{l}\text { Gesticulando na direção de ou } \\
\text { tocando a parte do corpo que } \\
\text { dói }\end{array}$ & $\begin{array}{l}\text { Tocando ou gesticulando em } \\
\text { direção ao membro que dói }\end{array}$ & $\begin{array}{l}\text { Gesticulando na direção de ou } \\
\text { tocando a parte do corpo que dói }\end{array}$ \\
\hline 19 & $\begin{array}{l}\text { Protecting, favoring or guarding } \\
\text { part of the body that hurts }\end{array}$ & $\begin{array}{l}\text { Protegendo, favorecendo ou } \\
\text { defendendo a parte do corpo } \\
\text { que dói }\end{array}$ & $\begin{array}{l}\text { Protegendo, favorecendo ou } \\
\text { defendendo a parte do corpo que } \\
\text { dói }\end{array}$ & $\begin{array}{l}\text { Protegendo, favorecendo ou } \\
\text { defendendo a parte do corpo que } \\
\text { dói }\end{array}$ \\
\hline 20 & $\begin{array}{l}\text { Flinching or moving the body part } \\
\text { away, being sensitive to touch }\end{array}$ & $\begin{array}{l}\text { Retraindo ou afastando a parte } \\
\text { do corpo, sendo sensível ao } \\
\text { toque }\end{array}$ & $\begin{array}{l}\text { Encolhendo ou recolhendo a parte } \\
\text { do corpo que se encontra sensível } \\
\text { ao toque }\end{array}$ & $\begin{array}{l}\text { Retraindo ou afastando a parte do } \\
\text { corpo, sendo sensível ao toque. }\end{array}$ \\
\hline 21 & $\begin{array}{l}\text { Moving the body in a specific way } \\
\text { to show pain (e.g., head back, } \\
\text { arms down, curls up, etc.) }\end{array}$ & $\begin{array}{l}\text { Movendo o corpo de maneira } \\
\text { específica para demostrar dor (p. } \\
\text { ex.: cabeça para trás, braços para } \\
\text { baixo, em posição fetal, etc.) }\end{array}$ & $\begin{array}{l}\text { Mexendo o corpo de uma maneira } \\
\text { específica para demonstrar dor (p. } \\
\text { ex., cabeça para trás, braços para } \\
\text { baixo, encurvar-se, etc.) }\end{array}$ & $\begin{array}{l}\text { Movendo o corpo de maneira espe- } \\
\text { cífica para demostrar dor (p. ex.: } \\
\text { cabeça para trás, braços para baixo, } \\
\text { em posição fetal, encolhido, etc.) }\end{array}$ \\
\hline 22 & Shivering & Tremor & Tremendo & Tremor \\
\hline 23 & Change in color, pallor & Alteração na cor, palidez & Alteração de cor, palidez & Alteração na cor, palidez \\
\hline 24 & Sweating, perspiring & Sudorese, transpiração & Suando, transpirando & Sudorese, transpiração \\
\hline 25 & Tears & Lágrimas & Lágrimas & Lágrimas \\
\hline 26 & Sharp intake of breath, gasping & Inspiração brusca, suspiro & Inspiração forçada, suspirando & Inspiração brusca, suspiro \\
\hline 27 & Breath holding & Prendendo a respiração & Apneia, prendendo a respiração & Prendendo a respiração \\
\hline
\end{tabular}

\section{Discussão}

O NCCPC-PV tem chamado a atenção de vários centros especializados no tratamento de pacientes com CCIC, tendo em vista sua fácil aplicabilidade, pois são necessários apenas 10 minutos de observação para completar os 27 itens, não sendo obrigatório estar continuamente olhando para o paciente (o respondente e o paciente devem apenas estar no mesmo ambiente), podendo ser usado tanto em ambientes hospitalares quanto em locais similares na vigência de dor aguda. $\mathrm{O}$ instrumento apresentou boa consistência e confiabilidade em vários estudos de validação. ${ }^{11,14-16}$ 
Tabela 2 Descrição da fase de retradução da versão T1,2

\begin{tabular}{|c|c|c|c|c|}
\hline & $\mathrm{T} 1,2$ & Retradução RT1 & Retradução RT2 & Versão RT1,2 \\
\hline 1 & $\begin{array}{l}\text { Gemendo, choramingando, } \\
\text { soluçando (levemente) }\end{array}$ & $\begin{array}{l}\text { Moaning, whimpering, sobbing } \\
\text { (slightly) }\end{array}$ & $\begin{array}{l}\text { Moaning, whimpering, sobbing } \\
\text { (slightly) }\end{array}$ & $\begin{array}{l}\text { Moaning, whimpering, sobbing } \\
\text { (slightly) }\end{array}$ \\
\hline 2 & Chorando (moderadamente alto) & Crying (moderately loud) & Crying (quite loud) & Crying (quite loud) \\
\hline 3 & Chorando/berrando (muito alto) & Crying/screaming (very loud) & Crying/yelling (very loud) & Crying/screaming (very loud) \\
\hline 4 & $\begin{array}{l}\text { Um som ou palavra específica } \\
\text { para a dor ( } p \text {. ex., choro ou tipo } \\
\text { de risada) }\end{array}$ & $\begin{array}{l}\text { A sound or specific word for the } \\
\text { pain (e.g., crying or a type of } \\
\text { laugh) }\end{array}$ & $\begin{array}{l}\text { A specific sound or word for the } \\
\text { pain (e.g., a cry or type of laugh) }\end{array}$ & $\begin{array}{l}\text { A specific sound or word for the } \\
\text { pain (e.g., a cry or type of laugh) }\end{array}$ \\
\hline 5 & $\begin{array}{l}\text { Não cooperativa, mal-humor- } \\
\text { ada, irritadiça, triste }\end{array}$ & $\begin{array}{l}\text { Uncooperative, grumpy, irritable, } \\
\text { sad }\end{array}$ & $\begin{array}{l}\text { Uncooperative, bad-tempered, } \\
\text { sad }\end{array}$ & $\begin{array}{l}\text { Uncooperative, bad-tempered, } \\
\text { irritable, sad }\end{array}$ \\
\hline 6 & $\begin{array}{l}\text { Menos interativa com os outros, } \\
\text { retraída }\end{array}$ & $\begin{array}{l}\text { Less interactive with others, } \\
\text { withdrawn }\end{array}$ & $\begin{array}{l}\text { Less interactive with others, } \\
\text { withdrawn }\end{array}$ & $\begin{array}{l}\text { Less interactive with others, } \\
\text { withdrawn }\end{array}$ \\
\hline 7 & $\begin{array}{l}\text { Buscando conforto ou } \\
\text { proximidade física }\end{array}$ & $\begin{array}{l}\text { Seeking comfort or physical } \\
\text { proximity }\end{array}$ & $\begin{array}{l}\text { Seeking comfort or physical } \\
\text { proximity }\end{array}$ & $\begin{array}{l}\text { Seeking comfort or physical } \\
\text { proximity }\end{array}$ \\
\hline 8 & $\begin{array}{l}\text { Difícil de distrair, incapaz de ser } \\
\text { satisfeita ou acalmada }\end{array}$ & $\begin{array}{l}\text { Hard to distract, unable to be } \\
\text { satisfied or calmed down }\end{array}$ & $\begin{array}{l}\text { Difficult to distract, incapable of } \\
\text { being satisfied or soothed }\end{array}$ & $\begin{array}{l}\text { Difficult to distract, incapable of } \\
\text { being satisfied or soothed }\end{array}$ \\
\hline 9 & Testa franzida & Frowning & Frowning & Frowning \\
\hline 10 & $\begin{array}{l}\text { Uma alteração nos olhos, } \\
\text { incluindo: olhos estrábicos, olhos } \\
\text { arregalados, olhos franzidos }\end{array}$ & $\begin{array}{l}\text { A change in the eyes, including: } \\
\text { cross-eyed, wide-eyed, squinting }\end{array}$ & $\begin{array}{l}\text { A change in the eyes, including: } \\
\text { squinting, wide eyes, ??? eyes (N.B. } \\
\text { The Portuguese in this line is } \\
\text { incomplete!) }\end{array}$ & $\begin{array}{l}\text { A change in the eyes, including: } \\
\text { cross-eyed, wide-eyed, squinting }\end{array}$ \\
\hline 11 & $\begin{array}{l}\text { Virando a boca para baixo, sem } \\
\text { sorrir }\end{array}$ & Mouth turned down, unsmiling & $\begin{array}{l}\text { Mouth turned down without } \\
\text { smiling }\end{array}$ & Mouth turned down, unsmiling \\
\hline 12 & $\begin{array}{l}\text { Lábios cerrados, apertados, } \\
\text { fazendo biquinho ou tremendo }\end{array}$ & $\begin{array}{l}\text { Lips closed, closed tight, pouting } \\
\text { or trembling }\end{array}$ & $\begin{array}{l}\text { Tightly-closed, pouting or trem- } \\
\text { bling lips }\end{array}$ & $\begin{array}{l}\text { Closed, tightly-closed, pouting or } \\
\text { trembling lips }\end{array}$ \\
\hline 13 & $\begin{array}{l}\text { Dentes cerrados ou rangendo, } \\
\text { mordendo ou pondo a língua } \\
\text { para fora }\end{array}$ & $\begin{array}{l}\text { Clenched or grinding teeth, biting } \\
\text { or sticking tongue out }\end{array}$ & $\begin{array}{l}\text { Tightly-closed or gritting teeth, } \\
\text { biting or sticking tongue out }\end{array}$ & $\begin{array}{l}\text { Clenched or gritting teeth, biting } \\
\text { or sticking tongue out }\end{array}$ \\
\hline 14 & $\begin{array}{l}\text { Sem se movimentar, menos } \\
\text { ativa, quieta }\end{array}$ & Not moving, less active, quiet & Motionless, less active, quiet & Motionless, less active, quiet \\
\hline 15 & Debatendo-se, agitada, inquieta & Struggling, agitated, restless & Struggling, agitated, restless & Struggling, agitated, restless \\
\hline 16 & Flácido & Flaccid & Flaccid & Flaccid \\
\hline 17 & Teso, espástico, tenso, rígido & Stiff, spastic, tense, rigid & Stiff, spastic, tense, rigid & Stiff, spastic, tense, rigid \\
\hline 18 & $\begin{array}{l}\text { Gesticulando na direção de ou } \\
\text { tocando a parte do corpo que } \\
\text { dói }\end{array}$ & $\begin{array}{l}\text { Gesturing toward or touching the } \\
\text { body part that hurts }\end{array}$ & $\begin{array}{l}\text { Gesticulating in the direction of, or } \\
\text { touching, the part of the body that } \\
\text { hurts }\end{array}$ & $\begin{array}{l}\text { Gesturing in the direction of, or } \\
\text { touching, the part of the body that } \\
\text { hurts }\end{array}$ \\
\hline 19 & $\begin{array}{l}\text { Protegendo, favorecendo ou } \\
\text { defendendo a parte do corpo } \\
\text { que dói }\end{array}$ & $\begin{array}{l}\text { Protecting, favoring or defending } \\
\text { the part of the body that hurts }\end{array}$ & $\begin{array}{l}\text { Protecting, favoring or defending } \\
\text { the part of the body that hurts }\end{array}$ & $\begin{array}{l}\text { Protecting, favoring or defending } \\
\text { the part of the body that hurts }\end{array}$ \\
\hline 20 & $\begin{array}{l}\text { Retraindo ou afastando a parte } \\
\text { do corpo, sendo sensível ao } \\
\text { toque }\end{array}$ & $\begin{array}{l}\text { Shrinking back or moving the part } \\
\text { of the body away, being sensitive } \\
\text { to touch }\end{array}$ & $\begin{array}{l}\text { Retracting or drawing back the } \\
\text { part of the body that is sensitive to } \\
\text { touch }\end{array}$ & $\begin{array}{l}\text { Shrinking back or moving the part } \\
\text { of the body away, being sensitive } \\
\text { to touch }\end{array}$ \\
\hline 21 & $\begin{array}{l}\text { Movendo o corpo de maneira } \\
\text { específica para demostrar dor } \\
\text { (p. ex.: cabeça para trás, braços } \\
\text { para baixo, em posição fetal, } \\
\text { encolhido, etc.) }\end{array}$ & $\begin{array}{l}\text { Moving the body in a specific way } \\
\text { to demonstrate pain (e.g.: head } \\
\text { back, arms down, adopting a fetal } \\
\text { position, etc.) }\end{array}$ & $\begin{array}{l}\text { Moving his/her body in a specific } \\
\text { way to demonstrate pain }\end{array}$ & $\begin{array}{l}\text { Moving his/her body in a specific } \\
\text { way to demonstrate pain (e.g.: } \\
\text { head back, arms down, adopting } \\
\text { a fetal position, etc.) }\end{array}$ \\
\hline 22 & Tremor & Tremor & Tremors & Tremor \\
\hline 23 & Alteração na cor, palidez & Change in color, paleness & Color change, paleness & Color change, paleness \\
\hline 24 & Sudorese, transpiração & Sweating, perspiration & Sweating, transpiring & Sweating, perspiring \\
\hline 25 & Lágrimas & Tears & Tears & Tears \\
\hline 26 & Inspiração brusca, suspiro & Sudden inspiration, sighing) & Sudden breathing, sighing & Sudden inhalation, sighing \\
\hline 27 & Prendendo a respiração & Holding their breath & Holding his/her breath & Holding his/her breath \\
\hline
\end{tabular}

Escolheu-se o processo de adaptação de Beaton et a ${ }^{13}$ por ele ser considerado consistente e detalhado para uma melhor adequação dos termos traduzidos para a língua alvo, no caso, o português falado no Brasil.

Uma limitação encontrada na amostra dos cuidadores foi que somente $40 \%$ de suas crianças de referência já tinham passado por experiências pós operatórias, por serem provenientes de serviço de atendimento médico em nível secundário o que pode ter provocado um viés na amostra. Apesar da possibilidade de esse fator ser limitante em relação à capacidade dos cuidadores de testar os itens do NCCPC-PV, essa amostra foi aceita, pois esse instrumento foi 
Tabela 3 Características clínicas das crianças de referência $(n=20)$

\begin{tabular}{|c|c|c|c|}
\hline \multicolumn{2}{|l|}{ Características } & \multirow[t]{2}{*}{$n$} & \multirow[t]{2}{*}{$\%$} \\
\hline Idade & $\begin{array}{l}3,09 \text { anos* } \\
30-41 \text { meses }\end{array}$ & & \\
\hline Diagnóstico & $\begin{array}{l}\text { Paralisia cerebral } \\
\text { Síndrome de Down } \\
\text { Microcefalia } \\
\text { Incontinência } \\
\text { Cornelia Lange } \\
\text { Pigmentar } \\
\text { Mielomeningocele }\end{array}$ & $\begin{array}{l}9 \\
4 \\
4 \\
1 \\
1 \\
1\end{array}$ & $\begin{array}{l}45 \\
20 \\
20 \\
5 \\
5 \\
5\end{array}$ \\
\hline Tratamento atual & $\begin{array}{l}\text { Fisioterapia } \\
\text { Fonoaudiologia } \\
\text { Terapia ocupacional } \\
\text { Hidroterapia } \\
\text { Equoterapia }\end{array}$ & $\begin{array}{l}20 \\
20 \\
20 \\
1 \\
1\end{array}$ & $\begin{array}{l}100 \\
100 \\
100 \\
5 \\
5\end{array}$ \\
\hline Internações prévias & $0,0^{*}$ & & \\
\hline Cirurgias prévias & $0,0^{*}$ & & \\
\hline Motivos & $\begin{array}{l}\text { Sem cirurgia } \\
\text { Hérnia inguinal } \\
\text { Cirurgia cardíaca } \\
\text { Troca de válvula de DVP } \\
\text { Lábio leporino } \\
\text { Gastrostomia } \\
\text { Cisto liquórico } \\
\text { Criptorquidia } \\
\text { Mudança de válvula } \\
\text { de derivação } \\
\text { ventrículo-peritoneal } \\
\text { Instalação de DVP }\end{array}$ & $\begin{array}{l}12 \\
3 \\
3 \\
1 \\
1 \\
1 \\
1 \\
1 \\
1 \\
1\end{array}$ & $\begin{array}{l}60 \\
15 \\
15 \\
5 \\
5 \\
5 \\
5 \\
5 \\
5\end{array}$ \\
\hline
\end{tabular}

Notas: " $m e d i a n a ; ~ "$ intervalo interquartil (IIQ).

Tabela 4 Características demográficas dos profissionais de saúde $(n=20)$

\begin{tabular}{|l|l|l|l|}
\hline Características & & $\boldsymbol{n}$ & $\%$ \\
\hline Profissão & Médico ortopedista & 3 & 15 \\
& Médico pediatra & 3 & 15 \\
& Médico clínico da dor & 2 & 10 \\
& Médico residente & 0 & 0 \\
& Enfermeiro & 3 & 15 \\
& Técnico/Auxiliar & 2 & 10 \\
& de enfermagem & & \\
& Terapeuta ocupacional & 3 & 15 \\
& Fisioterapeuta & 4 & 20 \\
\hline Local onde tem & Hospital público & 14 & 70 \\
contato com & Hospital privado & 2 & 10 \\
pacientes com & Ambulatório público & 7 & 35 \\
comprometimento & Ambulatório privado & 5 & 25 \\
cognitivo e incapacidade & & & \\
de comunicação & & & \\
\hline Tempo de experiência & $<1$ ano & 1 & 5 \\
com pacientes com & $1-5$ anos & 3 & 15 \\
comprometimento & $5-10$ anos & 2 & 10 \\
cognitivo e incapacidade & $10-20$ anos & 6 & 30 \\
de comunicação & $>20$ anos & 8 & 40 \\
\hline Tempo de formado & $<1$ ano & 0 & 0 \\
& $1-5$ anos & 0 & 0 \\
& $5-10$ anos & 0 & 0 \\
& $10-20$ anos & 10 & 50 \\
& $>20$ anos & 10 & 50 \\
\hline \multirow{2}{*}{} & & \\
& &
\end{tabular}

Tabela 5 Características demográficas dos cuidadores $(n=20)$

\begin{tabular}{|l|l|l|l|}
\hline Características & & $\boldsymbol{n}$ & $\%$ \\
\hline Parentesco & Mãe & 17 & 85 \\
& Pai & 1 & 5 \\
& Tia & 2 & 10 \\
\hline Quantidade diária & $6-12$ horas & 1 & 5 \\
de tempo & $12-18$ horas & 7 & 35 \\
passada com o & >18 horas & 11 & 55 \\
paciente & Dias alternados & 1 & 5 \\
\hline Profissão & "Do lar" & 15 & 75 \\
& Porteiro & 1 & 5 \\
& Cabeleireiro & 1 & 5 \\
& Técnico em enfermagem & 1 & 5 \\
& Administrador de empresa & 1 & 5 \\
& Odontólogo & 1 & 5 \\
\hline
\end{tabular}

desenvolvido não somente para dor pós-operatória, como também para outras situações de dor aguda, experiência comum entre tais crianças. Além do mais, a amostra testada não foi de pacientes mas de cuidadores com profundo conhecimento SOBRE O comportamento de seus cuidados, inclusive quanto à dor aguda. Assim, foi considerado como principal fator nos critérios de inclusão que os cuidadores tivessem experiência com crianças com CCIC, mesmo que essas não tenham passado por experiências pósoperatórias.

Quanto ao perfil, a maioria dos cuidadores era do sexo feminino (95\%), mães das crianças cuidadas (85\%), que dedicavam mais de 12 horas diárias à função de cuidadora (90\%), e que tinham o ambiente doméstico como principal local de atividade diária (75\%). Todos esses fatores estão relacionados, e demonstram o grande impacto que a DI exerce em suas famílias. Essa quase exclusiva participação do sexo feminino, principalmente de mães como cuidadoras, seguiu a mesma tendência da literatura. ${ }^{17,18}$

Para quantificar o nível de experiência de nossa amostra de profissionais de saúde, analisamos o tempo de experiência deles na prática de suas atividades profissionais. Observamos que todos os profissionais (100\%) tinham mais de 10 anos de formados em suas profissões. Com esse objetivo, também verificou-se que 14 profissionais (70\%) tinham mais de 10 anos de experiência no atendimento a pacientes com CCIC. Essa amostra foi considerada experiente em relação a suas atividades profissionais de saúde, colaborando com a boa qualidade da adaptação. Já a idade, apesar de não mostrar diferença significativa, revelou uma diferença de 10 anos entre cuidadores e profissionais de saúde.

Toda a amostra $(n=40)$ teve quase todas as medianas conjuntas entre 4 e 5 (apenas uma mediana com 3,5), ou seja, os itens traduzidos foram considerados "claros" e "muito claros" no geral.

Ao final do processo, o instrumento Br-NCCPC-PV, adaptado e descrito na - Figura 2, foi considerado adequado para profissionais e cuidadores de referência para ser utilizado em pacientes com CCIC após validado, sendo bem compreendido. 
Tabela 6 Medianas e percentis após avaliação da clareza da versão pré-teste

\begin{tabular}{|c|c|c|c|c|c|c|c|}
\hline & \multirow[t]{2}{*}{ Tradução da versão pré-final } & \multicolumn{2}{|c|}{$\begin{array}{l}\text { Profissionais de } \\
\text { saúde }\end{array}$} & \multicolumn{2}{|c|}{ Cuidadores } & \multicolumn{2}{|l|}{ Todos } \\
\hline & & Mediana & Percentis & Mediana & Percentis & Mediana & Percentis \\
\hline 1 & Gemendo, choramingando, soluçando (levemente) & 5 & $3,25-5,0$ & 4 & $4,0-4,0$ & 4 & $4,0-5,0$ \\
\hline 2 & Chorando (moderadamente alto) & 5 & $4,0-5,0$ & 4 & $4,0-4,75$ & 4 & $4,0-5,0$ \\
\hline 3 & Chorando/berrando (muito alto) & 5 & $5,0-5,0$ & 4 & $4,0-5,0$ & 5 & $4,0-5,0$ \\
\hline 4 & $\begin{array}{l}\text { Um som ou palavra específica para a dor } \\
\text { (p. ex., choro ou tipo de risada) }\end{array}$ & 4,5 & $2,25-5,0$ & 4 & $2,25-4,0$ & 4 & $2,25-5,0$ \\
\hline 5 & Não cooperativa, mal-humorada, irritada, triste & 5 & $4,0-5,0$ & 4 & $4,0-4,75$ & 4 & $4,0-5,0$ \\
\hline 6 & Menos interativa com os outros, retraída & 5 & $4,0-5,0$ & 4 & $3,25-4,0$ & 4 & $4,0-5,0$ \\
\hline 7 & Buscando conforto ou proximidade física & 5 & $4,0-5,0$ & 4 & $3,0-4,0$ & 4 & $4,0-5,0$ \\
\hline 8 & Difícil de distrair, incapaz de ser satisfeita ou acalmada & 5 & $4,0-5,0$ & 4 & $3,25-4,0$ & 4 & $4,0-5,0$ \\
\hline 9 & Testa franzida & 5 & $4,0-5,0$ & 2 & $2,0-4,0$ & 4 & $2,0-5,0$ \\
\hline 10 & $\begin{array}{l}\text { Uma alteração nos olhos, incluindo: olhos } \\
\text { estrábicos, olhos arregalados, olhos franzidos }\end{array}$ & 5 & $4,0-5,0$ & 3,5 & $2,0-4,0$ & 4 & $2,25-5,0$ \\
\hline 11 & Virando a boca para baixo, sem sorrir & 4 & $2,25-5,0$ & 2 & $1,0-4,0$ & 3,5 & $2,0-4,75$ \\
\hline 12 & Lábios cerrados, apertados, fazendo biquinho ou tremendo & 5 & $4,0-5,0$ & 3,5 & $2,0-4,0$ & 4 & $3,0-5,0$ \\
\hline 13 & $\begin{array}{l}\text { Dentes cerrados ou rangendo, mordendo } \\
\text { ou pondo a língua para fora }\end{array}$ & 4,5 & $3,25-5,0$ & 4 & $2,0-4,0$ & 4 & $2,25-5,0$ \\
\hline 14 & Sem se movimentar, menos ativa, quieta & 5 & $3,25-5,0$ & 4 & $4,0-4,75$ & 4 & $4,0-5,0$ \\
\hline 15 & Debatendo-se, agitada, inquieta & 5 & $5,0-5,0$ & 4 & $4,0-5,0$ & 5 & $4,0-5,0$ \\
\hline 16 & Flácido & 5 & $3,25-5,0$ & 2 & $1,25-4,0$ & 4 & $2,0-5,0$ \\
\hline 17 & Teso, espástico, tenso, rígido & 5 & $5,0-5,0$ & 4 & $2,0-4,0$ & 4 & $3,25-5,0$ \\
\hline 18 & Gesticulando na direção ou tocando a parte do corpo que dói & 5 & $5,0-5,0$ & 4 & $2,0-4,0$ & 4 & $4,0-5,0$ \\
\hline 19 & $\begin{array}{l}\text { Protegendo, favorecendo ou defendendo a parte } \\
\text { do corpo que dói }\end{array}$ & 5 & $4,0-5,0$ & 4 & $2,0-4,0$ & 4 & $3,25-5,0$ \\
\hline 20 & $\begin{array}{l}\text { Retraindo ou afastando a parte do corpo, sendo } \\
\text { sensível ao toque }\end{array}$ & 5 & $4,25-5,0$ & 4 & $3,0-4,0$ & 4 & $4,0-5,0$ \\
\hline 21 & $\begin{array}{l}\text { Movendo o corpo de maneira específica para demostrar dor } \\
\text { (p. ex.: cabeça para trás, braços para baixo, em posição fetal, } \\
\text { corpo encolhido, etc.) }\end{array}$ & 5 & $4,0-5,0$ & 4 & $4,0-4,0$ & 4 & $4,0-5,0$ \\
\hline 22 & Tremor & 5 & $4,0-5,0$ & 4 & $3,25-5,0$ & 4,5 & $4,0-5,0$ \\
\hline 23 & Alteração na cor da pele, palidez & 5 & $4,0-5,0$ & 4 & $3,0-5,0$ & 4 & $3,25-5,0$ \\
\hline 24 & Sudorese, transpiração & 5 & $4,0-5,0$ & 2 & $1,0-4,0$ & 4 & $2,0-5,0$ \\
\hline 25 & Lágrimas & 5 & $4,0-5,0$ & 5 & $4,0-5,0$ & 5 & $4,0-5,0$ \\
\hline 26 & Inspiração brusca, suspiro & 5 & $3,25-5,0$ & 4 & $2,0-4,0$ & 4 & $3,0-5,0$ \\
\hline 27 & Prendendo a respiração & 5 & $3,0-5,0$ & 4 & $2,0-4,0$ & 4 & $3,0-5,0$ \\
\hline
\end{tabular}

Tabela 7 Comparação entre cuidadores e profissionais de saúde quanto a idade, sexo e grau de instrução

\begin{tabular}{|c|c|c|c|c|c|c|}
\hline & & \multicolumn{2}{|c|}{ Cuidadores } & \multicolumn{2}{|c|}{$\begin{array}{l}\text { Profissionais de } \\
\text { saúde }\end{array}$} & \multirow[t]{2}{*}{ Valor de $p$} \\
\hline \multicolumn{6}{|l|}{ Característica } & \\
\hline \multirow[t]{3}{*}{ Idade (anos) } & & \multicolumn{2}{|c|}{$33,5^{*}$} & \multicolumn{2}{|c|}{$43,5^{*}$} & \multirow[t]{3}{*}{$1,0^{\triangle}$} \\
\hline & & \multicolumn{2}{|c|}{$30-41^{\#}$} & \multicolumn{2}{|c|}{$40-52^{\#}$} & \\
\hline & & $n$ & $\%$ & $\bar{n}$ & $\%$ & \\
\hline Sexor & $\begin{array}{l}\text { Feminino } \\
\text { Masculino }\end{array}$ & $\begin{array}{l}19 \\
1\end{array}$ & $\begin{array}{l}95 \\
5\end{array}$ & $\begin{array}{l}18 \\
2\end{array}$ & $\begin{array}{l}90 \\
10\end{array}$ & 1,0 \\
\hline \multirow[t]{4}{*}{ Grau de instrução ${ }^{\Omega}$} & Nível Superior & 2 & 10 & 20 & 100 & 0,001 \\
\hline & Nível médio completo & 6 & 30 & 0 & 0 & \\
\hline & Nível fundamental & 9 & 45 & 0 & 0 & \\
\hline & Nível fundamental incompleto & 3 & 15 & 0 & 0 & \\
\hline
\end{tabular}

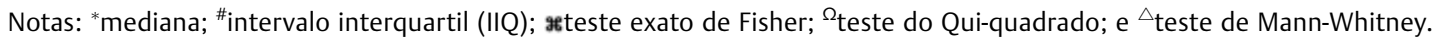


178 Tradução para português brasileiro e adaptação transcultural do NCCPC-PV Colares et al.

Lista de Verificação de Dor em Crianças Não Comunicantes - Versão Pós-Operatória
(Br-NCCPC-PV)
\begin{tabular}{|lll|}
\hline Nome: - & Unid./ Prontuário N & Data: \\
Observador: & Horário Inicial: & Horário Final: \\
\hline
\end{tabular}

Com que frequência essa criança tem mostrado estas reações nos últimos 10 minutos? Por favor, circule um número para cada reação. Caso um item não se aplique a essa criança (por exemplo, se a criança não consegue estender as mãos), então indique "não aplicável" para tal item.

0=NUNCASEM REAÇス̃o 1 = UM POUCO 2 = COM CERTA FREQUÊNCLA 3 = COM MUITA FREQUÊNCIA NA= NX̃o APLICÁVEL

\begin{tabular}{|c|c|c|c|c|c|c|}
\hline \multicolumn{7}{|c|}{ 1. Vocal } \\
\hline 1. & Gemendo, reclamando, choramingando (levemente)........... & 0 & 1 & 2 & 3 & NA \\
\hline 2. & 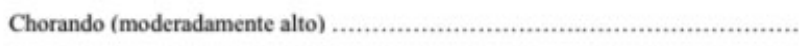 & $\mathbf{0}$ & 1 & 2 & 3 & NA \\
\hline 3. & Gritando/berrando (muito alto) & $\mathbf{0}$ & 1 & 2 & 3 & NA \\
\hline 4. & Um som ou palavra especifica para a dor (p. ex., choro ou tipo de risada) & $\mathbf{0}$ & 1 & 2 & 3 & NA \\
\hline
\end{tabular}

\begin{tabular}{|c|c|c|c|c|c|c|}
\hline \multicolumn{7}{|c|}{ II. Social } \\
\hline 5. & 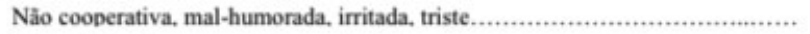 & 0 & 1 & 2 & 3 & NA \\
\hline 6. & Menos interativa com os outros, retraida ............. & 0 & 1 & 2 & 3 & NA \\
\hline 7. & Buscando conforto ou proximidade fisica ................................ & 0 & 1 & 2 & 3 & NA \\
\hline 8. & Dificil de distrair, incapaz de ser satisfeito ou acalmado ................................. & 0 & 1 & 2 & 3 & NA \\
\hline
\end{tabular}

\begin{tabular}{|c|c|c|c|c|c|c|}
\hline \multicolumn{7}{|c|}{ III. Facial } \\
\hline . & Testa franzida, com o rosto tenso..... & 0 & 1 & 2 & 3 & NA \\
\hline 10. & $\begin{array}{l}\text { Uma alteração nos olhos incluindo olhos apertados, olhos arregalados ou olhos } \\
\text { franzidos. }\end{array}$ & 0 & 1 & 2 & 3 & NA \\
\hline 11. & Virando a boca para baixo, sem sorrir, fazendo beicinho & $\mathbf{0}$ & 1 & 2 & 3 & NA \\
\hline 12. & Lábios cerrados, apertados, fazendo biquinho ou tremendo. & 0 & 1 & 2 & 3 & NA \\
\hline 13. & Dentes cerrados ou rangendo, mastigando ou pondo a lingua para fora $\ldots . . \ldots \ldots \ldots \ldots . . . .$. & 0 & 1 & 2 & 3 & NA \\
\hline
\end{tabular}

\begin{tabular}{|c|c|c|c|c|c|c|}
\hline \multicolumn{7}{|c|}{ IV. Atividade } \\
\hline 14. & Sem se movimentar, menos ativa, quieta. . & 0 & 1 & 2 & 3 & NA \\
\hline 15. & Debatendo-se, agitada, inquieta .. & 0 & 1 & 2 & 3 & NA \\
\hline
\end{tabular}

\begin{tabular}{|c|c|c|c|c|c|c|}
\hline \multicolumn{7}{|c|}{ V. Corpo e Membros } \\
\hline 16. & Flácido, com o corpo relaxado .. & $\mathbf{0}$ & 1 & 2 & 3 & NA \\
\hline 17. & Teso, espástico, tenso, rigido ... & $\mathbf{0}$ & 1 & 2 & 3 & NA \\
\hline 18. & Gesticulando na direção ou tocando a parte do corpo que dói & 0 & 1 & 2 & 3 & NA \\
\hline 19. & Protegendo, favorecendo ou defendendo a parte do corpo que dói .......................... & $\mathbf{0}$ & 1 & 2 & 3 & NA \\
\hline 20. & Encolhendo ou recolhendo a parte do corpo que encontra-se sensivel ao toque ........ & 0 & 1 & 2 & 3 & NA \\
\hline 21. & $\begin{array}{l}\text { Movendo o corpo de maneira especifica para demostrar dor (p. ex.: cabeça para trás, } \\
\text { braços para baixo, em posição fetal, corpo encolhido, etc.) }\end{array}$ & $\mathbf{0}$ & 1 & 2 & 3 & NA \\
\hline
\end{tabular}

\begin{tabular}{|c|c|c|c|c|c|c|}
\hline \multicolumn{7}{|c|}{ VI. Fisiológico } \\
\hline 22. & (2) & 0 & 1 & 2 & 3 & NA \\
\hline 23. & Alteração na cor da pele, palidez ......................... & $\mathbf{0}$ & 1 & 2 & 3 & NA \\
\hline 24. & Sudorese, suando muito, transpirando...... & $\mathbf{0}$ & 1 & 2 & 3 & NA \\
\hline 25. & 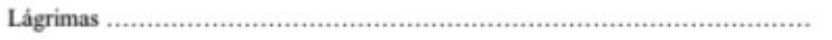 & $\mathbf{0}$ & 1 & 2 & 3 & NA \\
\hline 26. & Inspiração brusca, ofegante ... & 0 & 1 & 2 & 3 & NA \\
\hline 27. & Prendendo a respiração & 0 & 1 & 2 & 3 & NA \\
\hline
\end{tabular}

\section{Resumo da PONTUAÇão:}

\begin{tabular}{|c|c|c|c|c|c|c|c|}
\hline Categoria & I & II & III & IV & V & VI & TOTAL \\
\hline Pontuação: & & & & & & & \\
\hline
\end{tabular}

Versão 01.2004 $\bigcirc 2004$ Lynn Breau, Patrick McGrath, Allen Finley, Carol Camfield

Fig. 2 Lista de verificação de dor em crianças não comunicantes - versão pós-operatória (Br-NCCPC-PV). 


\section{USANDO O Br-NCCPC-PV}

A NCCPC-PV foi criada para ser usada em crianças com idade de 3 a 18 anos que sejam incapazes de falar por causa de dificuldades ou deficiências cognitivas (mentais/intelectuais). Pode ser usada em crianças com ou sem incapacidades ou deficiências físicas. As descriçø̃es dos tipos de crianças usadas para validar a NCCPC-PV podem ser encontradas em: Breau, L.M., Finley, G.A., McGrath, P.J. \& Camfield, C.S. (2002). Validation of the Non-Communicating Children's Pain Checklist - Postoperative Version. Apesthesiology, 96 (3), 528-535. A NCCPC-PV foi criada para ser usada sem treinamento por pais e cuidadores, ou por outros adultos que não tenham familiaridade com uma criança específica (não a conheçam bem).

A NCCPC-PV pode ser livremente copiada para uso clínico ou em pesquisas financiadas por entidades sem fins lucrativos. Entidades com fins lucrativos devem entrar em contato com Lynn Breau: Pediatric Pain Research, IWK Health Centre, 5850 University Avenue, Halifax, Nova Scotia Canada, B3J 3G9 (lbreau@ns.sympatico.ca).

A NCCPC-PV foi criada com a intenção de ser usada para dor após uma cirurgia ou devido a outros procedimentos conduzidos em uma unidade hospitalar que provoquem dor. Em caso de suspeita de dor de curta ou longa duração em crianças em domicilio ou em condições de cuidados domiciliares a longo prazo, a Lista de Verificação de Dor em Crianças NÃo COMUNICANTES - Revisada (NCCPC-R) pode ser usada. Pode ser obtida entrando-se em contato com Lynn Breau. Informações sobre o NCCPC-R podem ser encontradas em: Breau, L.M., McGrath, P.J., Camfield, C.S. \& Finley, G.A. (2002). Psychometric Properties of the Non-sommunicating Children's Rain Checklist-Revised. Pain, 99, 349-357.

\section{APLICAÇÃO:}

Para preencher a NCCPC-R, baseie suas observações no comportamento da criança durante 10 minutos. Não é necessário vigiar a criança continuamente durante esse periodo. Contudo, recomenda-se que o observador esteja na presença da criança durante a maior parte do tempo (p. ex.: esteja no mesmo cômodo que a criança). Embora períodos de observação mais curtos possam ser usados, as pontuações de corte descritas abaixo podem não ser aplicáveis.

Ao final do tempo de observação, indique com que frequência cada item foi visto ou ouvido. Isso não deve se basear no comportamento típico da criança ou em relação ao que ele(a) geralmente faz. Um guia para se decidir a frequência dos itens encontra-se abaixo:

\begin{tabular}{|c|c|c|}
\hline 0 & $=$ & $\begin{array}{l}\text { Não apresentado de forma alguma durante o período de observação. (importante, se o item não foi } \\
\text { observado porque a criança não foi capaz de executa-lo, deve-se marcá-lo como "NA"). }\end{array}$ \\
\hline 1 & $=$ & Visto ou ouvido raramente (quase nunca), porém presente. \\
\hline 2 & $=$ & Visto ou ouvido algumas vezes, mas não de forma contínua (não o tempo todo). \\
\hline 3 & $=$ & $\begin{array}{l}\text { Visto ou ouvido com frequência, quase continuamente (quase o tempo todo); qualquer um perceberia } \\
\text { isso facilmente caso visse a criança por poucos instantes durante este periodo de observação }\end{array}$ \\
\hline NA & $=$ & Não aplicável. Esta criança não é capaz de executar essa ação. \\
\hline
\end{tabular}

\section{PONTUAÇÃO:}

1. Some as pontuações para cada sub-escala e insira abaixo o número dessa sub-escala no Resumo da Pontuação ao final da tabela. Itens marcados "NA" recebem pontuação "0" (zero).

2. Some todas as pontuações das sub-escalas para obter a Pontuação Total.

3. Verifique se a pontuação da criança é maior do que a pontuação de corte.

\section{NOTA DE CORTE:}

Com base nas pontuações de 24 crianças com idades de 3 a 18 anos (Breau, Finley, McGrath \& Camfield, 2002), uma Pontuação Total de 11 ou mais indica que a criança tem dor moderada a severa. Com base em dados não publicados dessa mesma amostra, uma Pontuação total de 6-10 indica que a criança tem dor leve. Quando os pais e cuidadores preencheram a NCCPC-PV no hospital para o grupo de estudos, o mesmo mostrou-se preciso em $88 \%$ dos casos. Quando outros observadores preencheram a NCCPC-PV, o mesmo foi preciso em $75 \%$ dos casos. Uma pontuação total de 10 ou menos indica menos que uma dor moderada/severa. Isso estava correto no grupo de estudo para pais e cuidadores em $81 \%$ das vezes, e para outros observadores em $63 \%$ das vezes.

\section{USO DAS NOTAS DE CORTE:}

Como ocorre com toda ferramenta de observação, deve-se ter cuidado ao usar as pontuações de corte, pois elas podem não ser $100 \%$ precisas. Elas não devem ser usadas como a única base para se decidir se uma criança deve receber tratamento para dor. Em alguns casos, as crianças podem ter pontuações mais baixas quando há dor. Para instruções de uso mais detalhadas do NCCPC-PV em tais situações, por favor, consulte o manual completo, disponibilizado por Lynn Breau:

Fig. 2 (Continued) 
180 Tradução para português brasileiro e adaptação transcultural do NCCPC-PV Colares et al.

\section{Conclusão}

Este estudo adaptou o NCCPC-PV para o português falado no Brasil a fim de possibilitar sua melhor compreensão quando aplicado a cuidadores e profissionais de saúde ao medir dor aguda em crianças brasileiras. Depois de todo o processo, o Br-NCCPC-PV, adaptado à população brasileira, será validado em pacientes brasileiros para avaliar sua consistência interna e externa, testando sua confiabilidade.

\section{Conflito de Interesses}

Os autores declaram não haver conflito de interesses.

\section{Referências}

1 World Health Organization. Atlas. Global Resources for Persons with Intellectual Disabilities: 2007. World Health Organization; 2007:108

2 Maulik PK, Mascarenhas MN, Mathers CD, Dua T, Saxena S. Prevalence of intellectual disability: a meta-analysis of population-based studies. Res Dev Disabil 2011;32(02):419-436

3 Petterson B, Bourke J, Leonard H, Jacoby P, Bower C. Co-occurrence of birth defects and intellectual disability. Paediatr Perinat Epidemiol 2007;21(01):65-75

4 van Schrojenstein Lantman-De Valk HM, Metsemakers JF, Haveman MJ, Crebolder HF. Health problems in people with intellectual disability in general practice: a comparative study. Fam Pract 2000;17(05):405-407

5 Harbour CK, Maulik PK. Epidemiology of Intellectual Disability. Int Encycl Rehabil 2013;2010:1-7

6 LaChapelle DL, Hadjistavropoulos T, Craig KD. Pain measurement in persons with intellectual disabilities. Clin J Pain 1999;15(01):13-23

7 Breau LM, Camfield CS, McGrath PJ, Finley GA. The incidence of pain in children with severe cognitive impairments. Arch Pediatr Adolesc Med 2003;157(12):1219-1226
8 Oberlander TF, Grunau RE, Fitzgerald C, Whitfield MF. Does parenchymal brain injury affect biobehavioral pain responses in very low birth weight infants at 32 weeks' postconceptional age? Pediatrics 2002;110(03):570-576

9 Breau LM, Camfield CS, McGrath PJ, Finley GA. Risk factors for pain in children with severe cognitive impairments. Dev Med Child Neurol 2004;46(06):364-371

10 McGrath PJ, Rosmus C, Canfield C, Campbell MA, Hennigar A Behaviours caregivers use to determine pain in non-verbal, cognitively impaired individuals. Dev Med Child Neurol 1998; 40(05):340-343

11 Breau LM, Finley GA, McGrath PJ, Camfield CS, Ph D. Validation of the Non-communicating Children's Pain Checklist-Postoperative Version. Anesthesiology 2002;96(03):528-535

12 Guillemin F, Bombardier C, Beaton D. Cross-cultural adaptation of health-related quality of life measures: literature review and proposed guidelines. J Clin Epidemiol 1993;46(12):1417-1432

13 Beaton DE, Bombardier C, Guillemin F, Ferraz MB. Guidelines for the process of cross-cultural adaptation of self-report measures. Spine 2000;25(24):3186-3191

14 Johansson M, Carlberg EB, Jylli L. Validity and reliability of a Swedish version of the Non-Communicating Children's Pain Checklist-Postoperative Version. Acta Paediatr 2010;99(06): 929-933

15 Zanchi C, Massaro M, Ferrara G, et al. Validation of the Italian version of the Non-Communicating Children's Pain ChecklistPostoperative Version. Ital J Pediatr 2017;43(01):75

16 Zabalia M, Breau LM, Wood C, et al. Validation francophone de la grille d'évaluation de la douleur-déficience intellectuelle - version postopératoire. Can J Anaesth 2011;58(11):1016-1023

17 Pimenta RA. Avaliação da qualidade de vida e sobrecarga de cuidadores de pessoas com deficiência intelectual. Rev Bras Ciênc Saúde 2010;14(03):69-76

18 Pisula E, Porębowicz-Dörsmann A. Family functioning, parenting stress and quality of life in mothers and fathers of Polish children with high functioning autism or Asperger syndrome. PLoS One 2017;12(10):e0186536 